\title{
O edentulismo no Brasil: epidemiologia, rede assistencial e produção de próteses pelo Sistema Único de Saúde.
}

\section{Edentulism in Brazil: epidemiology, health care network, and prosthodontic production by the Unified Health System.}

\section{Edentulismo en Brasil: epidemiología, red de atención de salud y producción de prótesis por el Sistema Único de Salud.}

Erica Tatiane da SILVA ${ }^{1}$ Rommel Teodoro de OLIVEIRA ${ }^{2}$ Cláudio Rodrigues LELES ${ }^{3}$

Endereço completo para correspondência:

* Erica Tatiane da Silva. Fundação Oswaldo Cruz, FIOCRUZ - Unidade II - Brasília. Avenida L3 Norte, Campus Universitário Darcy Ribeiro, Gleba A, SC 4. Asa Norte. CEP: 70910900. Brasília, DF - Brasil. Telefone: (61) 33294602. E-mail: ericatatiane@fiocruz.br.

RESUMO: O objetivo do estudo foi analisar a conjuntura nacional quanto ao perfil epidemiológico do edentulismo, rede de atenção à saúde bucal e produção de próteses no âmbito do Sistema Único de Saúde. Foram utilizados dados epidemiológicos do Projeto SBBrasil 2010 e investigada a distribuição das Equipes de Saúde Bucal (ESB), Centros de Especialidades Odontológicas (CEOs), Laboratórios Regionais de Próteses Dentárias (LRPDs) e tratamento protético no SUS. Foram elaborados mapas para investigação da distribuição geográfica de variáveis do estudo e realizadas análises descritivas e correlacionais. A prevalência de perda dentária foi alta, especialmente entre mulheres, idosos e indivíduos de baixo nível de escolaridade e renda familiar, com destaque da região Norte. As necessidades de prótese foram superiores nas regiões Norte e Nordeste. Houve correlação entre o número de CEOs por município com tamanho populacional ( $\mathrm{p}<0,001 ; \mathrm{r}=0,473)$, índice de desenvolvimento humano ( $\mathrm{p}<0,001 ; \mathrm{r}=0,165)$ e número de $\mathrm{ESB}(\mathrm{p}<0,001 ; \mathrm{r}=0,403)$. As produções laboratoriais e ambulatoriais foram superiores nas regiões Sudeste, Sul e Nordeste. Apesar dos avanços proporcionados pela Política Nacional de Saúde Bucal, permanece um quadro de alta prevalência de perda dentária, necessidade de tratamento protético e desigualdades na oferta dos servicos.

1 Fundação Oswaldo Cruz, Brasília, Distrito Federal, Brasil.

2 Instituto de Informática, Universidade Federal de Goiás, Goiânia, Goiás, Brasil

3 Faculdade de Odontologia da Universidade Federal de Goiás, Goiânia, Goiás, Brasil 
Palavras-chave: Perda de dente; Prótese Dentária; Serviços de Saúde Bucal; Sistema Único de Saúde

ABSTRACT: This study aims to investigate epidemiologic aspects of edentulism, health care network, and prosthodontic production by the Brazilian Unified Health System. Data sets were taken from the following sources: 2010 Brazilian National Oral Health Survey (SBB10), Specialized Dental Care Centers (SDCCs), Regional Dental Prosthesis Laboratories (RDPLs), and prosthodontic treatment productivity by the public health system. Spatial analysis and descriptive and correlational statistics were performed. The prevalence of tooth loss was high, especially among women, elderly, individuals with low educational level and family income, and in the North region. Prosthetic needs were higher in the North and Northeast regions. The number of SDCCs by city was correlated with population size $(\mathrm{p}<0.001 ; \mathrm{r}=0.473)$, local Human Development Index $(p<0.001 ; r=0.165)$ and number of Oral Health Teams $(p<0.001 ; r=0.403)$. Laboratory and ambulatory productions were higher in the Southeast, South and Northeast regions. Despite the advances obtained with the Brazilian National Oral Health Policy, edentulism remains with high prevalence, prosthodontic treatment needs, and inequalities in service provision.

Keywords: Tooth Loss; Dental Prosthesis; Dental Health Services; Unified Health System

RESUMEN : El presente estudio tiene como objetivo investigar los aspectos epidemiológicos del edentulismo, red de atención de salud y la producción de prótesis por el Sistema Único de Salud de Brasil. Los datos fueron obtenidos de las siguientes fuentes: Encuesta Nacional de Salud Bucal Brasil 2010, Centros de Atención Dental Especializados (CADEs), de los Laboratorios Regionales de Prótesis Dental (LRPDs) y la productividad de tratamiento protésico por el sistema de salud pública. Se realizaron análisis espacial, descriptiva y correlacional. La prevalencia de pérdida de dientes fue alta, especialmente entre las mujeres, los ancianos, las personas con bajo nivel educativo y de ingresos de la familia, y en la región Norte. Necesidades protésicas fueron mayores en las regiones Norte y Nordeste. El número de CADEs por ciudad se correlaciona con el tamaño de la población $(\mathrm{p}<0,001 ; \mathrm{r}=0,473)$, índice de desarrollo humano local $(\mathrm{p}<0,001 ; \mathrm{r}=0,165)$ y número de Equipos de Salud Oral $(\mathrm{p}<0,001 ; \mathrm{r}=0,403)$. Laboratorio y ambulatorios producciones fueron mayores en las regiones Sur, Sudeste y Nordeste. A pesar de los avances obtenidos con la Política Nacional de Salud Bucal en Brasil, el panorama de la pérdida de dientes continúa con una alta prevalencia, necesidades de producción de prótesis, y desigualdades en la prestación de servicios. Palabras clave: Pérdida de Diente; Prótesis Dental; Servicios de Salud Dental; Sistema Único de Salud 


\section{INTRODUÇÃO}

O edentulismo é um problema de saúde pública que reflete a gravidade das condições de saúde bucal de determinada população, suas características socioculturais e o modelo de prática odontológica hegemônico. Sua alta prevalência em certos grupos populacionais e os consequentes impactos funcionais e estéticos que afetam negativamente a qualidade de vida desafiam os profissionais e os serviços de saúde a superarem esse cenário através de estratégias efetivas de prevenção e tratamento. ${ }^{1}$

No Brasil, paralelamente aos avanços técnico-científicos em odontologia, ainda coexistem a exclusão social e dívida assistencial, revelando deficiências no modelo de oferta de serviços em incorporar com resolutividade ações de promoção de saúde, prevenção e manejo das doenças e agravos em saúde bucal. Historicamente, o sistema público de saúde se caracterizou pela oferta reduzida de ações de baixa complexidade, predominantemente curativas e mutiladoras. ${ }^{2} \mathrm{~A}$ inserção da Equipe de Saúde Bucal (ESB) na Estratégia Saúde da Família (ESF) ocorreu no ano de 2001, contudo permaneciam as limitações quanto aos procedimentos de maior complexidade, ainda sem diretrizes específicas e incentivos federais. ${ }^{3,4}$

Em 2004, foi lançada a Política Nacional de Saúde Bucal (PNSB), fundamentada nos princípios de universalidade, equidade e integralidade. Entre suas principais linhas de ação, estão a ampliação e qualificação da atenção básica no âmbito da ESF e da atenção especializada. ${ }^{5,6}$ Com isso, os recursos financeiros destinados à saúde bucal aumentaram progressivamente, inclusive para tratamentos de média complexidade, através da implantação e custeio dos Centros de Especialidades Odontológicas (CEOs) e dos Laboratórios Regionais de Próteses Dentárias (LRPDs). ${ }^{3,4}$

Refletindo a atualidade desse processo de reorganização dos serviços de saúde bucal, são escassas as pesquisas voltadas à atenção secundária em odontologia no Sistema Único de Saúde (SUS), ${ }^{7-9}$ ainda mais sobre o tratamento protético. Os estudos têm focado no desenvolvimento da PNSB na perspectiva do financiamento federal ${ }^{3,4} \mathrm{e}$ dos fatores associados à acessibilidade e integralidade do cuidado, ${ }^{5,6}$ na avaliação dos $\operatorname{CEOs}^{7,8,10}$ e na evolução da produção de procedimentos odontológicos. ${ }^{9,11}$

Diante da importância de se investigar o contexto da atenção à saúde bucal para o enfrentamento do edentulismo, este trabalho teve como objetivo analisar a conjuntura nacional referente ao perfil epidemiológico desse agravo, rede assistencial e produção de próteses dentárias no âmbito do SUS.

\section{METODOLOGIA}

Foi delineado um estudo transversal com base em dados secundários sobre o perfil epidemiológico do edentulismo no Brasil, a distribuição da rede assistencial e a produção de próteses dentárias pelo 
serviço público. Para uma análise integrada desse cenário, o recorte temporal teve como referência o ano de 2010 - correspondente à realização do último levantamento epidemiológico nacional em saúde bucal (Projeto SBBrasil 2010), cujo banco de dados ${ }^{12}$ subsidiou a investigação dos aspectos epidemiológicos do edentulismo.

No SBBrasil 2010, foi investigado o uso e necessidade de prótese dentária nos grupos etários de 15 a 19, 35 a 44 e 65 a 74 anos, os quais são referência para avaliação das condições de saúde bucal em adolescentes, adultos e idosos, respectivamente. A necessidade normativa de tratamento protético foi determinada conforme avaliação dos examinadores quanto à presença de espaço protético e qualidade insatisfatória da prótese no que se refere à retenção, estabilidade e reciprocidade, fixação e/ou estética. Já a necessidade subjetiva foi obtida por meio de entrevista abordando a autopercepção dos indivíduos sobre a necessidade de uso ou substituição de sua prótese. ${ }^{13}$

Para investigação da perda dentária, foram utilizados os dados obtidos na avaliação de cárie dentária e necessidade de tratamento, considerando-se os dentes perdidos por cárie ou outra razão. Foram classificados como desdentados totais aqueles com ausência de todos os elementos dentários e como desdentados parciais os indivíduos com pelo menos um dente ausente, excluindo-se os terceiros molares. Estes últimos foram estratificados de acordo com o número de dentes presentes para identificação de desdentados com dentição funcional (presença de menos 20 dentes).

A partir desses dados, foi analisada a prevalência do edentulismo, uso e necessidade de prótese para o Brasil e por Unidade da Federação (UF). O numerador compreendeu o número de indivíduos com a condição de interesse e o denominador correspondeu ao total de indivíduos nas faixas etárias avaliadas para o edentulismo - exceto para uso de prótese, em que os dados do SBBrasil foram obtidos apenas para os indivíduos desdentados.

Para identificação da rede pública de atenção secundária quanto à distribuição geográfica do número de CEOs e dos municípios com LRPDs credenciados até dezembro de 2010, foram coletados dados do sítio da Coordenação Nacional de Saúde Bucal (CNSB) do Departamento de Atenção Básica $(\mathrm{DAB})^{14}$.

Investigou-se, também, a presença de ESB implementadas em cada município - conforme dados do sítio do DAB ${ }^{15}$ o Índice de Desenvolvimento Humano Municipal (IDH-M) - utilizando como fonte o Atlas de Desenvolvimento Humano no Brasil; ${ }^{16}$ e a população residente em 2010 obtida do censo demográfico do Instituto Brasileiro de Geografia e Estatística. ${ }^{17}$

Por fim, foram analisados a produção e os gastos com próteses dentárias pelo SUS, empregandose o banco de dados do Sistema de Informação Ambulatorial do SUS (SIA/SUS), ${ }^{17}$ segundo a produção por local de atendimento no período de 2001 (ano do início da inserção da ESB na ESF) 
a 2010 (considerando o horizonte temporal em análise).

Para cada UF, foram obtidos as quantidades e os valores aprovados para pagamento pelas Secretarias de Saúde, considerando os seguintes procedimentos e seus códigos de correspondência na Tabela de Procedimentos, Medicamentos, Órteses e Próteses e Materiais Especiais do SUS: i) prótese parcial removível (PPR) - códigos 10.083.02-2, 10.083.03-0, 10.083.04-9, 07.01.07.009-9 e 07.01.07.010-2; ii) prótese total (PT) - códigos 10.082.10-7, 10.082.11-5, 10.082.13-1, 10.082.14-0, 07.01.07.012-9 e 07.01.07.013-7; iii) prótese parcial fixa (PPF) - códigos 10.082.04-2, 10.084.02$9,10.084 .03-7,10.084 .04-5,10.084 .05-3,10.084 .06-1,10.084 .07-0,10.084 .08-8$ e $07.01 .07 .014-$ 5; e iv) prótese dentária sobre implante (PSI) - código 07.01.07.075-3. Também foram incluídos os procedimentos ambulatoriais referentes à moldagem dento-gengival, instalação e adaptação da prótese (códigos 03.051.01-3, 03.07.04.003-8 e 03.07.04.007-0) e ao reembasamento e conserto de prótese dentária (códigos 10.081.04-6 e 03.07.04.008-9).

Foram realizadas análises descritivas e inferenciais com emprego do Stata 12.0 (Stata Corp., College Station, Estados Unidos). Para os dados do Projeto SBBrasil, a correção pelo efeito de desenho foi feita usando-se o comando svy para a análise de dados oriundos de amostras complexas. O TabWin 3.6b (Datasus, Brasil) foi utilizado para elaboração de mapas com apresentação da prevalência de edentulismo, uso e necessidade de prótese, e da produção de prótese por UF conforme os tercis de distribuição de cada variável -, bem como da rede de atenção à saúde bucal por município - com círculo proporcional para o número de registros. Após verificação da normalidade da distribuição dos dados pelo teste de Kolmogorov-Smirnov, realizou-se correlação de Spearman entre o número de CEOs por município e o tamanho populacional, IDH-M e número de ESB, considerando-se um nível de significância de 5\%.

Este artigo faz parte de uma tese de Doutorado defendida junto ao Programa de Pós-graduação em Ciências da Saúde da Universidade Federal de Goiás. Como o estudo utilizou bases de dados secundárias e de domínio público, foi dispensado de apreciação por comitê de ética em pesquisa.

\section{RESULTADOS}

A prevalência de perda dentária nos indivíduos investigados para o edentulismo no Projeto SBBrasil $2010(\mathrm{n}=22.440)$ foi de 64,8\% (IC 95\%=63,1-66,5). Esse cenário foi mais crítico entre mulheres, idosos, indivíduos de baixo nível de escolaridade e renda familiar, com destaque para a Região Norte (Tabela 1). 
Tabela 1. Distribuição dos indivíduos examinados no SBBrasil segundo condição dentária em relação ao edentulismo, de acordo com características demográficas e socioeconômicas. Brasil, 2010.

\begin{tabular}{|c|c|c|c|c|c|}
\hline & \multirow{3}{*}{$\begin{array}{l}\text { Frequência } \\
\text { (\%) }\end{array}$} & \multicolumn{4}{|c|}{ Edentulismo - \% (IC 95\%)* } \\
\hline & & \multicolumn{3}{|c|}{ Sim } & \multirow[t]{2}{*}{ Não } \\
\hline & & $\begin{array}{c}\begin{array}{c}\text { Ausência total de } \\
\text { dentes }\end{array} \\
\end{array}$ & $\begin{array}{c}1 \text { a } 19 \text { dentes } \\
\text { presentes }\end{array}$ & $\begin{array}{c}\geq 20 \text { dentes } \\
\text { presentes }\end{array}$ & \\
\hline \multicolumn{6}{|l|}{ Sexo } \\
\hline Feminino & $13860(61,8)$ & $14,9(13,2-16,6)$ & $17,4(15,8-18,9)$ & $36,3(34,4-38,3)$ & $31,4(29,2-33,6)$ \\
\hline Masculino & $8580(38,2)$ & $11,2(9,3-13,2)$ & $13,0(11,4-14,6)$ & $35,2(31,8-38,6)$ & $40,6(37,1-44,0)$ \\
\hline \multicolumn{6}{|l|}{ Grupo etário } \\
\hline $15-19$ & $5367(23,9)$ & $* *$ & $* *$ & $19,3(16,7-21,9)$ & $80,5(77,8-83,1)$ \\
\hline $35-44$ & $9564(42,6)$ & $2,9(2,1-3,8)$ & $18,1(15,9-20,4)$ & $61,3(58,8-63,7)$ & $17,7(14,9-20,4)$ \\
\hline $65-74$ & $7509(33,5)$ & $53,9(49,6-58,1)$ & $33,8(30,8-36,9)$ & $11,5(9,3-13,7)$ & $0,8(0,2-1,3)$ \\
\hline \multicolumn{6}{|c|}{ Raça autorreferida } \\
\hline Branca & $9747(43,4)$ & $14,2(12,3-16,1)$ & $15,8(14,2-17,4)$ & $34,5(32,5-36,5)$ & $35,5(33,0-37,9)$ \\
\hline Parda & $9655(43,0)$ & $11,0(9,1-12,8)$ & $15,2(13,5-16,8)$ & $39,3(36,9-41,8)$ & $34,5(32,1-36,9)$ \\
\hline Preta & $2461(11,0)$ & $17,5(13,8-21,2)$ & $16,9(13,2-20,6)$ & $31,4(25,5-37,4)$ & $34,1(29,5-38,7)$ \\
\hline Amarela & $386(1,7)$ & $14,5(6,7-22,3)$ & $8,8(3,9-13,6)$ & $27,4(15,6-39,1)$ & $49,4(31,9-66,8)$ \\
\hline Indígena & $191(0,9)$ & $16,4(1,0-33,8)$ & $11,9(4,8-18,9)$ & $38,1(20,9-55,3)$ & $33,6(13,2-54,0)$ \\
\hline \multicolumn{6}{|c|}{ Escolaridade (anos) } \\
\hline 0 & $1332(6,0)$ & $59,0(52,1-65,8)$ & $25,5(19,4-31,6)$ & $13,5(6,8-20,3)$ & $2,0(0,7-3,3)$ \\
\hline $1-4$ & $4705(21,2)$ & $32,8(28,8-36,6)$ & $28,8(25,6-32,0)$ & $30,6(26,6-34,6)$ & $7,8(5,7-10,0)$ \\
\hline $5-8$ & $6020(27,2)$ & $10,8(7,8-13,9)$ & $16,2(14,3-18,1)$ & $39,8(36,2-43,4)$ & $33,2(29,3-37,0)$ \\
\hline $9-11$ & $6422(29,0)$ & $2,9(1,5-4,3)$ & $8,7(6,4-11,0)$ & $35,8(32,6-38,9)$ & $52,6(49,3-55,9)$ \\
\hline$\geq 12$ & $3687(16,6)$ & $2,2(1,1-3,3)$ & $8,1(5,9-10,3)$ & $42,0(38,0-46,1)$ & $47,7(42,7-52,5)$ \\
\hline \multicolumn{6}{|c|}{ Renda familiar ( $\mathrm{R} \$$ ) } \\
\hline Até 500 & $3087(14,3)$ & $14,8(11,2-18,3)$ & $16,9(13,6-20,2)$ & $34,7(31,0-38,3)$ & $33,6(29,2-38,0)$ \\
\hline $501-1.500$ & $11294(52,2)$ & $15,1(13,4-16,8)$ & $17,1(15,4-18,7)$ & $36,8(33,8-39,8)$ & $31,0(28,7-33,3)$ \\
\hline $1.501-2.500$ & $4035(18,7)$ & $11,7(8,8-14,6)$ & $13,6(11,3-15,9)$ & $33,0(27,6-38,4)$ & $41,7(35,5-47,8)$ \\
\hline $2.501-4.500$ & $2007(9,3)$ & $8,9(5,6-12,2)$ & $10,6(7,4-13,8)$ & $36,3(30,4-42,1)$ & $44,2(37,7-50,8)$ \\
\hline$\geq 4.501$ & $1202(5,6)$ & $2,6(1,1-4,1)$ & $12,6(7,4-17,9)$ & $40,5(29,9-51,1)$ & $44,2(34,1-54,3)$ \\
\hline \multicolumn{6}{|l|}{ Região } \\
\hline Sudeste & $3773(16,8)$ & $13,9(12,4-15,5)$ & $14,1(12,5-15,7)$ & $35,6(33,1-38,0)$ & $36,4(33,7-39,0)$ \\
\hline Sul & $3577(15,9)$ & $13,4(11,4-15,4)$ & $18,4(16,4-20,3)$ & $33,6(31,0-36,1)$ & $34,6(31,6-37,6)$ \\
\hline Centro-oeste & $3410(15,2)$ & $13,3(12,0-14,7)$ & $15,8(14,2-17,4)$ & $36,4(34,1-38,6)$ & $34,5(31,9-36,9)$ \\
\hline Nordeste & $6094(27,2)$ & $12,6(11,3-13,8)$ & $16,3(15,1-17,5)$ & $37,1(35,2-39,0)$ & $34,0(31,9-36,2)$ \\
\hline Norte & $5586(24,9)$ & $10,3(8,9-11,5)$ & $20,1(18,4-21,9)$ & $41,0(38,4-43,6)$ & $28,6(25,9-31,2)$ \\
\hline
\end{tabular}

IC95\%: intervalo de 95\% de confiança

* Corrigido para o desenho amostral

$* *$ Valor $<1 \%$

Fonte: SBBrasil $2010^{12}$

Entre aqueles com perda dentária ( $\mathrm{n}=14.541), 49,6 \%$ utilizavam algum tipo de prótese dentária. A necessidade de prótese determinada pelos examinadores do SBBrasil (49,7\%; IC95\%: 48,1-51,4) 
foi alta entre usuários de prótese (66,8\%; IC95\%: 65,6-68,5), mostrando-se superior àquela advinda da autopercepção dos participantes quanto à condição de saúde bucal (29,5\%; IC95\%: 28,1-30,9).

Foram observadas disparidades entre as UFs em relação ao edentulismo e ao uso e necessidade de prótese dentária (Figura 1). A prevalência do edentulismo foi maior na região Norte, enquanto o uso de prótese teve destaque na região Sul. Já as necessidades tratamento protético foram superiores nas regiões Norte e Nordeste, seguida da Centro-oeste.

Figura 1. Edentulismo, uso de prótese e necessidade normativa e subjetiva de tratamento protético por Unidade da Federação. Brasil, 2010.
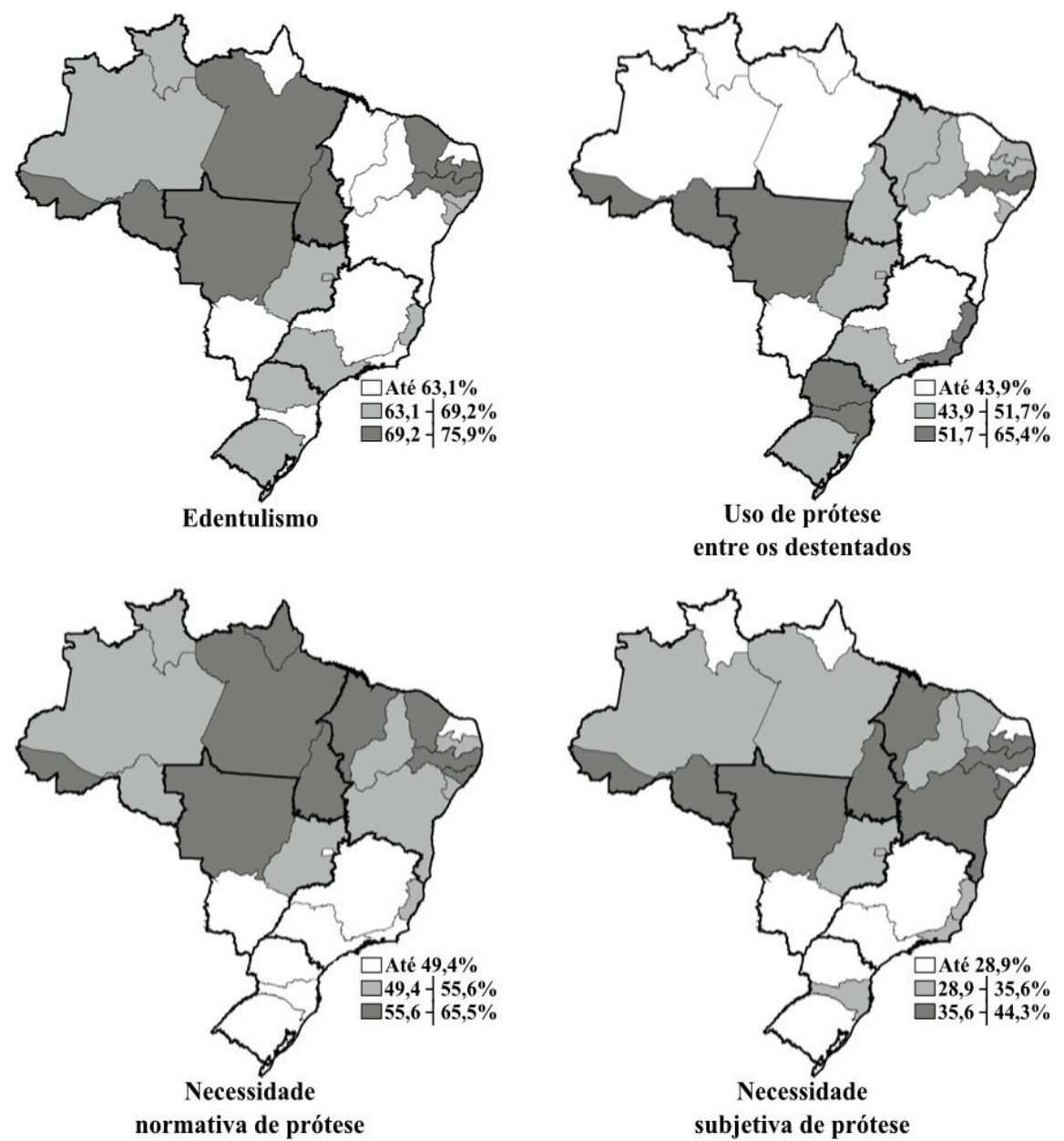

Fonte: SBBrasil $2010^{12}$

A Figura 2 apresenta a distribuição espacial da rede pública de atenção à saúde bucal no Brasil, revelando menor concentração de CEOs, LRPDs e ESB na região Norte. O número de CEOs por município correlacionou-se positivamente com o tamanho populacional ( $\mathrm{p}<0,001 ; \mathrm{r}=0,473)$, IDH-M ( $\mathrm{p}<0,001 ; \mathrm{r}=0,165)$ e número de ESB implantadas $(\mathrm{p}<0,001 ; \mathrm{r}=0,403)$. 
Figura 2. Rede de atenção à saúde bucal no âmbito do Sistema Único de Saúde. Brasil, 2010.

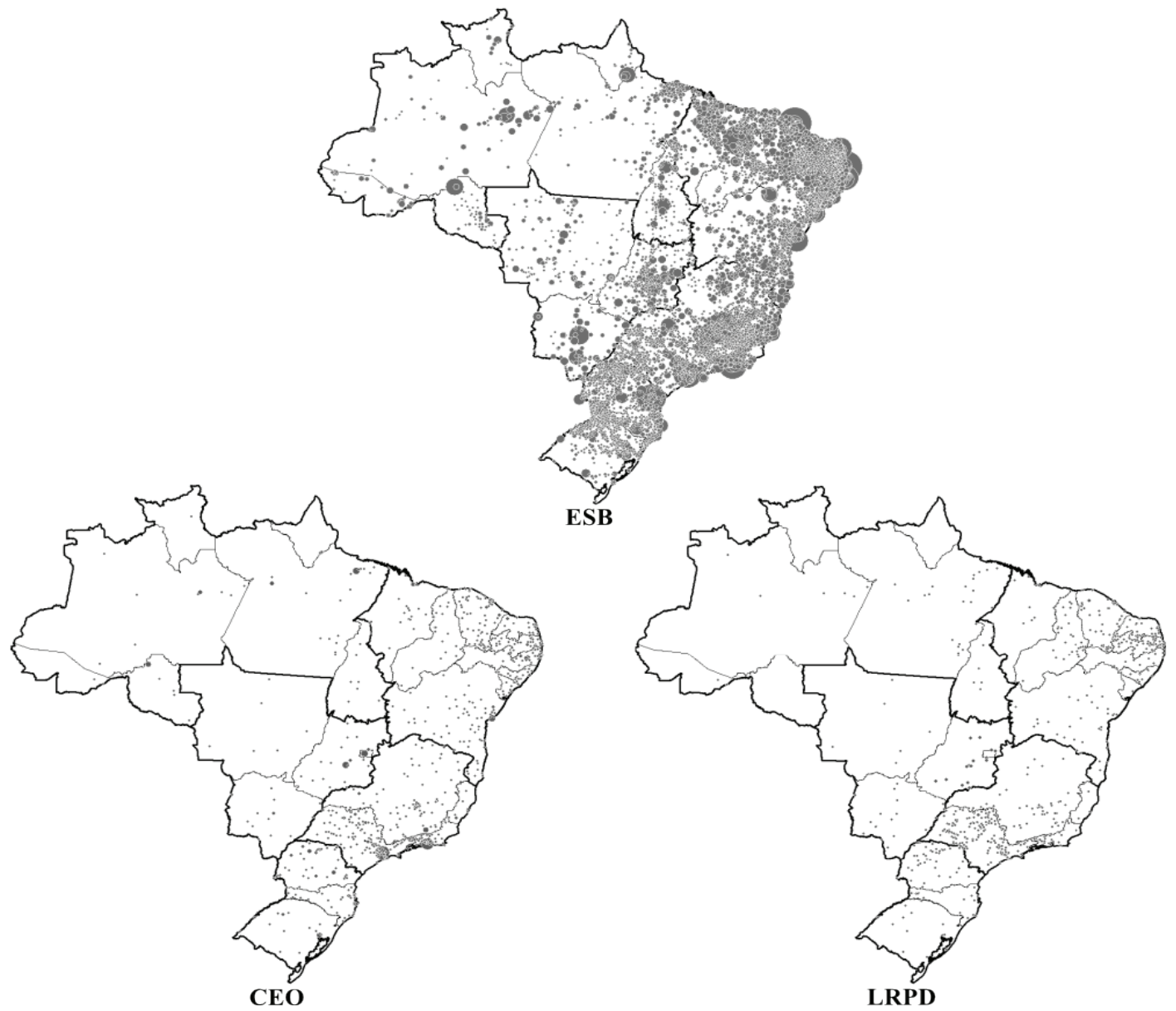

ESB: Equipe de Saúde Bucal; CEO: Centro de Especialidades Odontológicas; LRPD: Laboratório Regional de Prótese Dentária

Fonte: DAB ${ }^{14,15}$

Em relação à produção de próteses dentárias no âmbito do SUS, no período de 2001 a 2010, foram aprovados 1.103.818 procedimentos de confecção de prótese dentária: 64,7\% PT, 20,6\% PPF e 14,7\% PPF. Adicionalmente, houve 2.448.248 procedimentos de moldagem dento-gengival, instalação e adaptação de prótese dentária e 294.105 de reembasamento e conserto de prótese.

Os valores aprovados para a confecção de próteses e procedimentos ambulatoriais foram, respectivamente, de $\mathrm{R} \$ 16.700 .921,33$ e $\mathrm{R} \$ 253.422,88$ para a região Sudeste; $\mathrm{R} \$ 8.472 .227,19$ e R\$ 27.545,36 para a região Sul; R \$ 8.351.432,56 e R\$ 34.151,56 para a região Nordeste; R\$ 2.412.946,27 e R \$ 8.863,56 para a região Centro-Oeste; e $\mathrm{R} \$ 1.762 .744,33$ e R $\$ 16.781,72$ para a região Norte. A distribuição desses procedimentos por UF consta na Figura 3. De modo geral, o número de procedimentos foi superior nas regiões Sul, Sudeste e Nordeste e inferior no Norte. No Centro-oeste, houve destaque da produção ambulatorial. 
Figura 3. Distribuição geográfica da quantidade de procedimentos de prótese dentária aprovados pelo SUS. Brasil, 2001-2010.
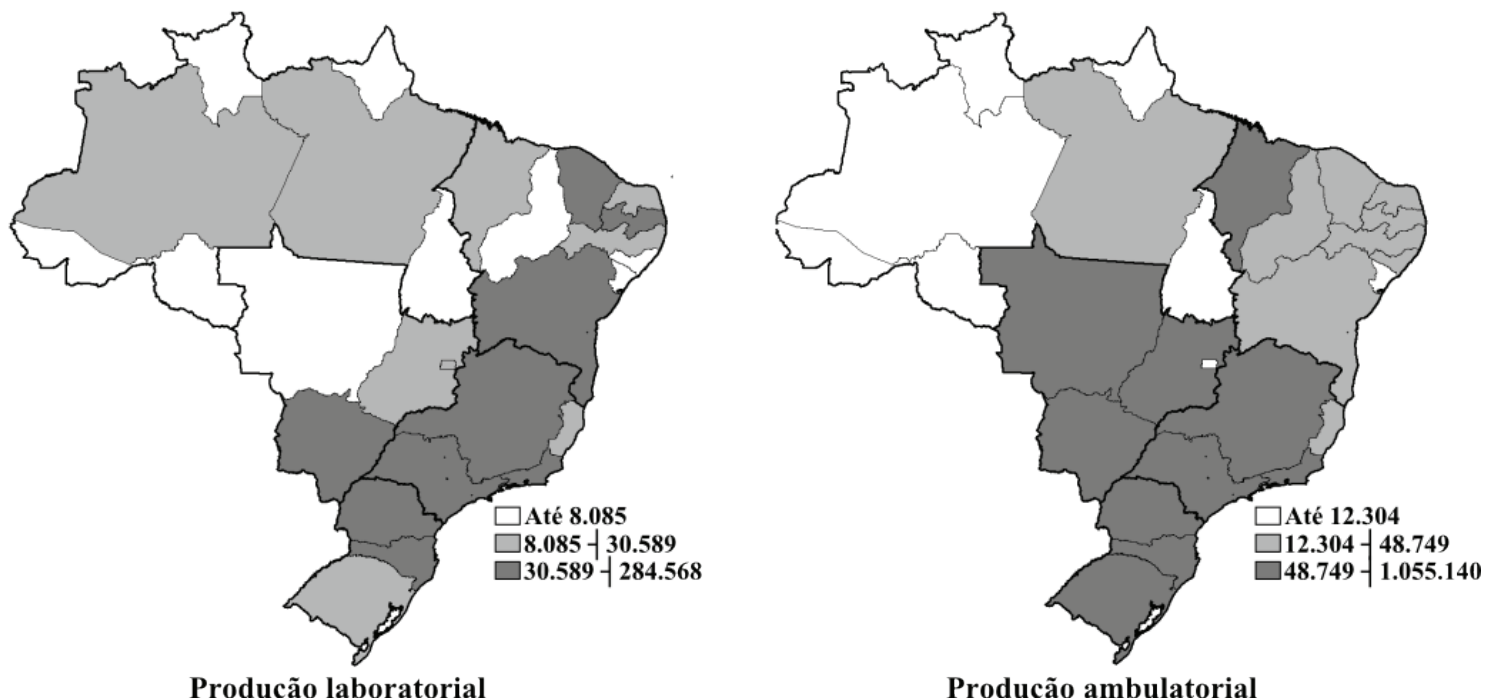

Fonte: SIA/SUS ${ }^{17}$

A análise conjunta da situação epidemiológica do edentulismo e do serviço reabilitador protético no âmbito do SUS evidenciou um quadro de alta prevalência de perda dentária e necessidade protética no Brasil, especialmente em indivíduos e regiões geográficas mais vulneráveis quanto à condição de saúde bucal e ao acesso e utilização dos serviços.

\section{DISCUSSÃO}

O presente trabalho fornece um panorama abrangente do edentulismo no Brasil, englobando desde aspectos epidemiológicos, à configuração da rede assistencial e produção de próteses dentárias no serviço público em âmbito nacional.

A prevalência de edentulismo é alta, especialmente em indivíduos mais velhos e residentes na região Norte (Tabela 1). Grande parcela dos indivíduos de 35 a 44 anos e de 65 a 74 anos apresentam perdas dentárias, corroborando dados da literatura nacional de que a maioria dos adultos desdentados é portador de dentição funcional, ${ }^{18-20}$ enquanto que a maioria dos idosos possui ausência de todos os dentes. ${ }^{18,19} \mathrm{O}$ aspecto cumulativo das duas principais doenças bucais (cárie e doença periodontal) faz com que sua sequela máxima, configurada pela perda dentária, seja mais prevalente nesses indivíduos. Além disso, por muito tempo, os serviços se pautaram na oferta de exodontias, o que é refletido nas condições bucais dos idosos de hoje. ${ }^{1,21,22}$ Apesar de ser menos prevalentes nos adolescentes, perdas dentárias nessa faixa etária destacam a dificuldade de manutenção dos dentes pelos serviços odontológicos, mesmo entre os mais jovens.

Conforme verificado na Figura 1, a demanda para reabilitação protética também permanece alta, principalmente no Norte e no Nordeste, ou seja, nas regiões brasileiras com menor IDH. 
Segundo o relatório do Projeto SBBrasil 2010, ${ }^{23}$ de 2003 para 2010, enquanto a situação do idoso se manteve, houve decréscimo considerável das necessidades de reabilitação protética entre os adolescentes (redução de 52\%) e adultos (redução de 70\%), porém 13,7\% e 68,8\% desses indivíduos ainda necessitam de algum tipo de prótese, respectivamente. Destaca-se que, apesar do número considerável de usuários de próteses, os resultados revelam uma necessidade de reparos ou substituições na maioria dos casos $(66,8 \%)$, reforçando a importância da ampliação da oferta de tratamento protético no SUS, além da proservação desses pacientes.

Para a necessidade de tratamento, chamam atenção as semelhanças entre os mapas que representam as necessidades normativa e subjetiva de prótese (Figura 1), alertando para a situação crítica vivenciada pelos indivíduos desdentados. Entretanto, a necessidade normativa se mostra superestimada quando comparada àquela percebida pelo paciente. Isso corrobora a relevância da inclusão de medidas subjetivas na tomada de decisão, visando reduzir o risco de sobretratamento e melhorar a relação entre custos e benefícios do tratamento. ${ }^{24,25}$

A maioria dos CEOs se localiza em municípios com ESB implantadas e LRPDs (Figura 2), em consonância com a não recomendação de sua implantação em locais onde a atenção básica não se encontra bem estruturada (geralmente em municípios menores e menos desenvolvidos), pois expõe a atenção secundária às pressões da livre demanda e execução de procedimentos típicos da atenção básica. ${ }^{7,8}$

Por outro lado, as diferenças geográficas no número de CEOs e LRPDs (Figura 2) suscitam preocupações sobre desigualdades na oferta e acesso aos serviços especializados, sobretudo em municípios com menor porte populacional e IDH-M.

Apesar da grande produção em prótese dentária, esses números ainda são incipientes frente às necessidades de tratamento. ${ }^{11,25}$ Há disparidades geográficas na quantidade de próteses confeccionadas e procedimentos ambulatoriais relacionados - e, consequentemente, dos respectivos valores aprovados -, com uma maior produção nos estados mais populosos do Brasil. Quando confrontada essa produção (Figura 3) com a necessidade de prótese (Figura 1), notase que é preciso expandir o tratamento protético no SUS, especialmente na região Norte e para as modalidades de próteses parciais. Não houve registro de prótese dentária sobre implante, refletindo a então recente publicação da Portaria 718/SAS de dezembro de 2010, por meio da qual o Ministério da Saúde passou a financiar novos procedimentos, incluindo o implante dentário osteointegrado.

O emprego de mapas permitiu uma análise integrada da distribuição espacial do edentulismo, uso e necessidade de prótese, da presença de ESB, CEOs e LRPDs, e da quantidade de procedimentos protéticos no SUS, evidenciando cenários mais críticos nas regiões Norte e Nordeste. Essas regiões 
correspondem àquelas com maiores proporções de indivíduos que nunca foram ao dentista, maior intervalo de tempo desde a última consulta e maior proporção de consultas motivadas pela dor. ${ }^{23}$

Embora o aumento dos investimentos em saúde bucal tenha reduzido as desigualdades no acesso e ampliado a utilização dos serviços odontológicos, as iniquidades permanecem expressivas. Além da oferta de tratamento protético, a influência de características estruturais, culturais e socioeconômicas podem repercutir em diferenças geográficas quanto ao uso dos serviços de saúde. ${ }^{25-27}$ Iniquidades sociais são associadas à maioria dos agravos bucais e ao acesso e utilização dos serviços em nível ecológico e individual. ${ }^{26-28}$ Somando-se a isso, se, por um lado, a diferença na alocação dos recursos entre as diferentes regiões e estados está em conformidade com a produção dos serviços, por outro, acaba sancionando desigualdades na distribuição da oferta dos serviços. ${ }^{29}$

Entre as limitações do estudo estão àquelas inerentes ao uso de dados secundários, particularmente para o SIA/SUS. Mesmo com realização de críticas e filtros contra inconsistências, há possibilidade de fraudes ou lançamentos incorretos, ou seja, a produção aprovada pode não corresponder à oferta de serviços realizados. ${ }^{30}$ Também não foram investigados a dinâmica da interface da atenção em saúde bucal, a relação entre oferta, acesso e utilização dos serviços, os fatores influenciadores do cumprimento das metas desses serviços e a qualidade da atenção. Ficam, então, evidenciadas áreas estratégicas para pesquisa. Tais estudos possibilitariam a proposição de estratégias de intervenção, incluindo critérios para ampliação da atenção especializada, melhoria da resolutividade dos serviços e implantação de protocolos de referência e contrarreferência.

\section{CONCLUSÃO}

Conclui-se que, mesmo com avanços significativos proporcionados pela PNSB, permanece um quadro de alta prevalência de perda dentária, necessidade de tratamento protético e desigualdades na oferta dos serviços. Diante disso, é necessário o desenvolvimento de parâmetros epidemiológicos e sócio-odontológicos para a orientação do planejamento dos serviços, associados a medidas para enfrentamento das desigualdades sociais e das iniquidades em saúde.

\section{REFERÊNCIAS BIBLIOGRÁFICAS}

1. Petersen PE. The World Oral Health Report 2003: continuous improvement of oral health in the 21 st century - the approach of the WHO Global Oral Health Programme. Community Dent Oral Epidemiol. 2003;Suppl:3-23.

2.Roncalli AG. A organização da demanda em serviços públicos de saúde bucal: universalidade, equidade e integralidade em saúde bucal coletiva [tese]. Araçatuba (SP): Universidade Estadual Paulista; 2000. 238 p. 
3. Maia LS, Kornis GEM. A reorganização da atenção à saúde bucal frente aos incentivos federais: a experiência fluminense. Rev APS. 2010;13(1):84-95.

4. Kornis GEM, Maia LS, Fortuna RFP. Evolução do financiamento da atenção à saúde bucal no SUS: uma análise do processo de reorganização assistencial frente aos incentivos federais. Rev Saúde Coletiva. 2001;21(1):197-215.

5. Chaves SCL, Barros SG, Cruz DN, Figueiredo ACL, Moura BLA, Cangussu MCT. Política Nacional de Saúde Bucal: fatores associados à integralidade do cuidado. Rev Saúde Pública. 2010;44(6):1005-13.

6. Souza LF, Chaves SCL. Política Nacional de Saúde Bucal: acessibilidade e utilização de serviços odontológicos especializados em um município de médio porte na Bahia. Rev Baiana Saúde Pública. 2010;34(2):371-87.

7. Figueiredo N, Goes PSA. Construção da atenção secundária em saúde bucal: um estudo sobre os Centros de Especialidades Odontológicas em Pernambuco, Brasil. Cad Saúde Pública. 2009;25(2):259-67.

8.Goes PSA, Figueiredo N, Neves JC, Silveira FMM, Costa JFR, Pucca Júnior JÁ, Rosales MS. Avaliação da atenção secundária em saúde bucal: uma investigação nos centros de especialidades do Brasil. Cad Saúde Pública. 2012;Suppl 28:S81-9.

9. Colussi CF, Calvo MCM, Nickel DA, Caetano JC, Silva ACB. Análise da evolução da produção de procedimentos odontológicos de média e alta complexidade na rede de serviços públicos em Santa Catarina. Cad Saúde Coletiva. 2009;17(4):939-50.

10. Chaves SCL, Cruz DN, Barros SG, Figueiredo AL. Avaliação da oferta e utilização de especialidades odontológicas em serviços públicos de atenção secundária na Bahia, Brasil. Cad Saúde Pública. 2011;27(1):143-54.

11. Azevedo TDR, Groisman S. Evolução dos procedimentos de próteses dentárias no SUS versus a necessidade instalada no sudeste do Brasil. Revista Científica do CRO-RJ. 2011;1(2):105.

12. Brasil. Ministério da Saúde. Departamento de Atenção Básica. Coordenação Geral de Saúde Bucal. Banco de dados da Pesquisa Nacional de Saúde Bucal - Projeto SBBrasil 2010. 2012 [capturado em 26 abri. 2012]. Disponível em: http://dab.saude.gov.br/CNSB/sbbrasi1/.

13. Brasil. Ministério da Saúde. Departamento de Atenção Básica. Coordenação Geral de Saúde Bucal. SBBrasil 2010: pesquisa nacional de saúde bucal: manual da equipe de campo. Brasília, 
DF: Ministério da Saúde, 2009.

14. Brasil. Ministério da Saúde. Departamento de Atenção Básica. Coordenação Geral de Saúde Bucal. Cidades atendidas. 2012 [capturado em 31 out. 2012]. Disponível em: http://dab.saude.gov. br/portaldab/ape_brasil_sorridente.php?conteudo=cidades_bs.

15. Brasil. Ministério da Saúde. Departamento de Atenção Básica. Evolução do credenciamento e implantação da estratégia Saúde da Família. 2015 [capturado em 02 nov. 2015]. Disponível em: http://dab.saude.gov.br/portaldab/historico_cobertura_sf.php.

16. Programa das Nações Unidas para o Desenvolvimento. Atlas de Desenvolvimento Humano no Brasil. 2015 [capturado em 02 nov. 2015] Disponível em: http://www.atlasbrasil.org.br/2013/.

17. Brasil. Ministério da Saúde. Departamento de Informática do SUS. Informações de Saúde. 2015 [capturado em 13 nov. 2015]. Disponível em: http://www.datasus.gov.br.

18. Peres MA, Barbato PR, Reis SCGB, Freitas CHSM, Antunes JLF. Perdas dentárias no Brasil: análise da Pesquisa Nacional de Saúde Bucal 2010. Rev Saúde Pública. 2013;Suppl 3:78-89.

19. Ribeiro MTF, Rosa MAC, Lima RMN, Vargas AMD, Haddad JPA, Ferreira EF. Edentulism and shortened dental arch in Brazilian elderly from the National Survey of Oral Health 2003. Rev Saúde Pública. 2011;45(5):817-23.

20. Koltermann AP, Giordani JMA, Pattussi MP. The association between individual and contextual factors and functional dentition status among adults in Rio Grande do Sul State, Brazil: a multilevel study. Cad Saúde Pública. 2011;27(1):173-82.

21. Owen CP, Locker D. Demographic, psychological, sociological, and economic variables and other factors that justify the need for prosthodontic services, and that help to assess the outcome of care. Int J Prosthodont. 2003;Suppl 16:19-26.

22. Carlsson GE, Omar R. The future of complete dentures in oral rehabilitation. A critical review. J Oral Rehabil. 2010;37(2):143-56.

23. Brasil. Ministério da Saúde. Departamento de Atenção Básica. Coordenação Geral de Saúde Bucal. Projeto SBBrasil 2010: Pesquisa Nacional de Saúde Bucal: resultados principais. Brasília, DF: Ministério da Saúde, 2011.

24. Leles CR, Freire MC. A sociodental approach in prosthodontic treatment decision making. J Appl Oral Sci. 2004;12(2):127-32. 
25. Aguiar VR, Celeste RK. Necessidade e alocação de laboratórios regionais de prótese dentária no Brasil: um estudo exploratório. Ciênc Saúde Coletiva. 2015;20(10): 3121-8.

26. Pinheiro RJ, Aguiar FP, Sass PE, Vilela MJ. Diferenças no uso de serviços odontológicos entre os estados do Brasil: uma análise baseada em modelos hierárquicos. Cad Saúde Coletiva. 2006;14(1):141-8.

27. Pinheiro RS, Torres TZG. Uso de serviço odontológico entre os estados do Brasil. Cienc Saúde Coletiva. 2006;11(4):999-1010.

28. Peres KG, Peres MA, Boing AF, Bertoldi AD, Bastos JL, Barros AJD. Redução das desigualdades sociais na utilização de serviços odontológicos no Brasil entre 1998 e 2008. Rev Saúde Pública. 2012;46(2):250-8.

29. Marques RM, Mendes A. Democracia, Saúde Pública e Universalidade: o difícil caminhar. Saúde Soc. 2007;16(3):35-51.

30. Barros SG, Chaves SLA. Utilização do Sistema de informações Ambulatoriais (SIASUS) com instrumento para caracterização das ações de saúde bucal. Epidemiol Serv Saúde. 2003;12(1):41-51.

Artigo apresentado em 05-01-14

Artigo aprovado em 21-08-15 Artigo publicado no sistema em 22-12-15 